\title{
Endogenous Soluble TRAIL Contributes to the Survival and Growth of Human Mesenchymal Stem Cells
}

\section{Joaquín Marco-Brualla ${ }^{1,2}$, Ana Gallego-Lleyda ${ }^{2}$, Jara Sanz ${ }^{1}$, Paula Desportes ${ }^{1}$, Pablo Fernández ${ }^{1}$, Luis Martínez-Lostao ${ }^{3}$, Pedro Lapuente ${ }^{1}$ and Alberto Ane ${ }^{2^{*}}$ (iD)}

${ }^{1}$ Living Cell/Fuenlabrada University Hospital (Peaches Biotech Group), Fuenlabrada, Spain

${ }^{2}$ Immunity, Cancer \& Stem Cells Group, Biochemistry and Molecular and Cell Biology Department, University of Zaragoza, Spain

${ }^{3}$ Immunology Department, Lozano Blesa Clinical Hospital/Aragón Health Research Institute, Spain

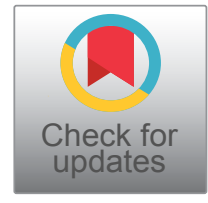

*Corresponding author: Prof. Alberto Anel, Ph.D, Immunity, Cancer \& Stem Cells Group, Biochemistry and Molecular and Cell Biology Department, University of Zaragoza, Pedro Cerbuna s/n, Zaragoza, 50009, Spain, Tel: 34-976761279, Fax: 34-976762123

\begin{abstract}
Background: TNF-related apoptosis-inducing ligand (TRAIL) is known to induce caspase-dependent apoptosis in tumor cells, while sparing normal cells. However, TRAIL is also able to induce proliferation in cells that are resistant to cell death induction, since it can also activate NF-kB-dependent signaling pathways. Human mesenchymal stem cells (hMSC) transfected with TRAIL have been used to treat cancer, with promising results in pre-clinical models, especially in gliomas. Regarding endogenous TRAIL expression in hMSC or its possible role in cell death, proliferation or differentiation, not much has been explored as yet.

Methods: Cell death induction by soluble TRAIL (sTRAIL) or by TRAIL associated with liposomes (LUV-TRAIL) on hMSC was studied by annexin-V-FITC and 7-AAD staining and flow cytometry. The effect of STRAIL, LUV-TRAIL or a blocking anti-TRAIL mAb on cell growth was studied by uptake of 5-bromo-2'-deoxyuridine (BrdU). Cell morphology after the treatments was studied by phase microscopy. Exosomes were separated from cell supernatant by sequential centrifugations and ultracentrifugation. Endogenous TRAIL expression in hMSC or in their derived exosomes was studied by immunoblot and flow cytometry.
\end{abstract}

Results: We demonstrated that exogenous TRAIL alone, either in soluble form or associated with liposomes (LUVTRAIL), does not affect growth of hMSC. In addition, it is also unable to induce cell death in these cells. Nevertheless, endogenous TRAIL from hMSC did affect positively their proliferation in combination with growth factors present in the serum. We demonstrated the expression of endogenous
TRAIL in hMSC, mainly with an intracellular distribution, and showed that it is secreted to the culture supernatant in soluble form, but not in association with exosomes, contributing in this way to hMSC viability and growth.

Conclusion: These observations could be important if hMSC transfected with TRAIL are used as tumor treatment or in regenerative medicine, since secreted TRAIL could exert its anti-tumoral effect at the same time as hMSC would persist.

\section{Keywords}

MSC, TRAIL, Exosomes, Liposomes

\begin{abstract}
Abbreviations
TRAIL: TNF-Related Apoptosis-Inducing Ligand; hMSC: Human Mesenchymal Stem Cells; LUV-TRAIL: Large Unilamellar Vesicles Coated with TRAIL; sTRAIL: Soluble TRAIL, FBS, Fetal Bovine Serum
\end{abstract}

\section{Introduction}

TNF-related apoptosis-inducing ligand (TRAIL) is a protein that belongs to the TNF superfamily. Since its discovery [1,2], its ability to induce tumor cell death has been demonstrated while sparing normal cells, inducing caspase-dependent apoptosis in a wide variety of tumor types [3] or necroptosis if caspases are inhibited [4]. TRAIL is able to recognize several transmembrane receptors, including TRAIL-R1 (DR4), TRAIL-R2 (DR5),

Citation: Marco-Brualla J, Gallego-Lleyda A, Sanz J, Desportes P, Fernández P, et al. (2021) Endogenous Soluble TRAIL Contributes to the Survival and Growth of Human Mesenchymal Stem Cells. Int J Stem Cell Res Ther 8:072. doi.org/10.23937/2469-570X/1410072

Accepted: March 11, 2021; Published: March 13, 2021

Copyright: (c) 2021 Marco-Brualla J, et al. This is an open-access article distributed under the terms of the Creative Commons Attribution License, which permits unrestricted use, distribution, and reproduction in any medium, provided the original author and source are credited. 
TRAIL-R3 (DcR1) and TRAIL-R4 (DcR2), as well as circulating osteoprotegerin. Among them, only DR4 and DR5 transduce TRAIL death signaling, while DcR1 and DcR2 act as decoy receptors as they lack functional death domains [5].

In order to exert its function, TRAIL homotrimerizes upon binding to its death receptors [6]. Following this event, the death domains of the oligomerized death receptors recruit the adapter protein Fas-associated death domain (FADD), which also induces the recruitment of procaspase- 8 and other proteins, such as the receptor-interacting serine/threonine-protein kinase 1 (RIPK1) and cellular inhibitor of apoptosis proteins (clAP's). This complex can promote caspase-8 activation, which is released to the cytosol and cleaves effector caspases-3 and -7, ultimately triggering apoptosis in the target cell [7].

However, TRAIL is also able to induce proliferation in cells that are resistant to cell death induction, since it can also activate NF-KB-dependent signaling pathways $[8,9]$. For instance, TRAIL enhances survival and proliferation in human vascular endothelial cells [10], erythroid differentiation [11], angiogenesis and neovascularization in microvascular endothelial cells [12] or promotes proliferation and survival [13]. In addition, TRAIL can also induce a cytoprotective autophagy through AMPK-dependent pathways [14].

In human mesenchymal stem cells (hMSC) it has been described that recombinant soluble TRAIL or transfection with TRAIL does not induce cell death, proliferation or differentiation, but induces their migration [15] TRAIL also enhances preadipocyte proliferation in a defined cellular model [16] and promotes an inflammatory response [17].

Apart from this, TRAIL has been extensively used in combination with MSC to treat cancer by injection of MSC transfected with TRAIL in growing tumors, with promising results in pre-clinical models. Most notably, this treatment has proved to reduce tumor burden in subcutaneous xenografts from several cancer types and lung metastasis in mice [18], colorectal carcinoma [19] and especially gliomas [20], being able to synergize with other compounds for a higher efficiency in tumor clearance $[21,22]$.

This anti-tumor effect could be mediated by TRAIL secretion either in soluble form or in association with exosomes. Physiologically, TRAIL is secreted from activated $T$ lymphocytes during an immune response in association with exosomes, which enhances its pro-apoptotic effects [23,24]. In fact, TRAIL-coated lipid nanoparticles (LUV-TRAIL) greatly increase in vitro cytotoxicity against many tumor cell types, compared to its soluble counterpart $[25,26]$. This property has been also demonstrated in vivo, at least for mammary and colon tumors $[27,28]$, and improved by encapsulating doxorubicin inside LUVTRAIL against especially resistant sarcoma cells [29].
On the other hand, MSC, as many other cells, secrete exosomes, which diverse content may have pleiotropic and therapeutic effects [30].

Regarding endogenous TRAIL expression in hMSC or its possible role in cell death, proliferation or differentiation, not much has been explored as yet. In this work, we isolated hMSC from adipose tissue from healthy donors and assessed the effect of soluble TRAIL or of LUV-TRAIL on them. We demonstrated that exogenous TRAIL does not affect growth, viability or differentiation of hMSC, either in soluble form or bound to liposomes. Nevertheless, endogenous TRAIL from hMSC, which was secreted in soluble form and not in association with exosomes, did affect positively their proliferation.

\section{Materials and Methods}

\section{Cells and cell culture}

Jurkat clone E6.1 (derived from human acute T cell leukemia) was obtained from ATCC and cultured in high glucose RPMI (GIBCO ${ }^{\mathrm{TM}}$ ) medium supplemented with $10 \%$ heat-inactivated FBS (Sigma), penicillin/streptomycin (Pan Biotech) and GlutaMAX (Gibco).

hMSC were freshly obtained at CRES-PhisiUP clinic, using previously optimized methods [31]. Briefly, abdominal fat was extracted from patients and washed three times with physiological serum (B. Braun). Supernatants were collected from these centrifugations and processed with collagenase (Lyposmol ADSC Enzyme ${ }^{\circ}$ ), which was previously reconstituted in Ringer-lactate (B. Braun). Samples were vigorously mixed for 2 minutes and then incubated at $37{ }^{\circ} \mathrm{C}, 150 \mathrm{rpm}$ for 45 minutes. Afterwards, collagenase was neutralized with equal amounts of Alpha-MEM Eagle medium (Lonza) supplemented with penicillin/streptomycin (Linus), stable glutamine (Biowest), amphotericin B (Linus) and 5\% Platelet Lysate, UltraGro (Helios) [Alpha MEM complete medium] and centrifuged $(700 \mathrm{~g}, 10 \mathrm{~min})$. Stromal vascular fraction was collected and incubated with cold 4 ${ }^{\circ} \mathrm{C}$ lysis buffer (Zenbio) (1:4 vol: vol) for 5 minutes, in order to eliminate erythrocytes. Samples were centrifuged $(570 \mathrm{~g}, 10 \mathrm{~min})$, followed by pellet resuspension in Alpha MEM complete medium, and cell suspension was seeded in culture flasks (TPP). Finally, the adherent fraction was refreshed next day with new Alpha MEM complete medium and used for experimentation.

Obtaining this biological material from voluntary donors or patients was made following Spanish and European law, upon signature of a written informed consent redacted and approved by the CRES Clinic in Zaragoza, Spain. Donors were also informed of the procedure, that is a registered mark of Peaches Biotech, termed "Lyposmol ADSC system". All procedures were performed in accordance with the Declaration of Helsinki guidelines.

Long-term culture of cells and treatments were performed in a biomed laboratory located outside of the 
clinic where samples from patients were obtained. Cells were cultured in different media, as indicated, at $37^{\circ} \mathrm{C}$ with $5 \% \mathrm{CO}_{2}$ in a cell incubator Heraeus Cell.

\section{Ultracentrifugation}

Aliquots $(60 \mathrm{ml})$ of supernatants from hMSC (4 confluent $150 \mathrm{~cm}^{2}$ culture flasks), Alpha MEM Eagle Medium alone, or Alpha MEM complete medium were obtained by centrifugation $(3000 \mathrm{~g}, 20 \mathrm{~min})$. Supernatants were then ultracentrifuged at $100000 \mathrm{~g}$ for $18 \mathrm{~h}$, as previously described [24]. Supernatants were collected and pellets were diluted in Alplha MEM Eagle Medium in $120 \mu \mathrm{l}$ for Western-Blot analysis.

\section{Generation of lipid vesicles coated with soluble recombinant TRAIL}

Preparation of large unilamellar vesicles (LUV) with anchored human soluble recombinant TRAIL (sTRAIL) was performed as previously described [25,32]. In brief, a mixture of phosphatidylcholine (PC), sphingomyelin (SM), cholesterol (CHOL), 1,2-distearoyl-sn-glycero-3-phosphoethanolamine)- $\mathrm{N}$-(methoxy(polyethylene glycol)-2000) (ammonium salt) (18:0 PEG2000-PE), and 1,2-dioleoyl-sn-glycero-3-\{[N-(5-amino-1-carboxypentyl)-iminodiacetic acid]succinyl\}x (nickel salt) (DOGSNTA-Ni) (all from Avanti Polar Lipids), was prepared in chloroform with a weight ratio of 50:30:10:5:5 and dried in two steps: First, under nitrogen gas; and second, under vacuum. Afterwards, lipids were resuspended in PBS, freeze-thawed 10 times and extruded 10 times through two polycarbonate membranes with a pore size of 200 $\mathrm{nm}$ (Whatman) using an extruder (Northern Lipids), in order to generate LUV. After that, LUV-TRAIL were obtained by incubation of LUV in PBS for $30 \mathrm{~min}$ at $37^{\circ} \mathrm{C}$ with sTRAlL-His ${ }_{6}$ (corresponding to amino acids 95-281, kindly provided by Dr. M. MacFarlane [33].

\section{Flow cytometry analysis}

For these assays, a FACSCalibur flow cytometer and CellQuestPro software (BD Biosciences) were used.

For the initial phenotyping of the cells, $1 \times 10^{5} \mathrm{hMSC}$ cells were incubated with FITC-conjugated $\mathrm{mAb}$ recognizing respectively CD45, CD34, CD44, CD73 or CD90 or with an isotype control (all from BD) in PBS containing $5 \% \mathrm{FBS}$ for $30 \mathrm{~min}$ at $4{ }^{\circ} \mathrm{C}$. Then, cells were washed, resuspended to $300 \mu \mathrm{l}$ with the corresponding buffer and analyzed by flow cytometry.

For the determination of surface expression of death receptors, $1 \times 10^{5} \mathrm{hMSC}$ cells were incubated with PE-conjugated anti-DR4, anti-DR5, anti-DcR1, anti-DcR2 monoclonal antibodies or isotype control (all from eBioscience) in the same way. For the determination of TRAIL expression on the surface of hMSC, the same labeling protocol was followed, using the anti-TRAIL mAb RIK-2 conjugated with PE (BD Pharmingen). For the determination of total TRAIL expression by flow cytometry, cells were previously fixed in $4 \%$ paraformaldehyde, permea- bilized with $0.1 \%$ saponin, and labeled in the same way.

For the measurement of cell death induction, $1 \times 10^{5}$ hMSC cells were stained for $15 \mathrm{~min}$ at room temperature simultaneously with Annexin-V-FITC (Immunostep), which binds to the phosphatidylserine exposed in the cell surface, and 7-aminoactinomycin D (7-AAD, Biolegend), which binds DNA, in annexin-binding buffer (140 $\mathrm{mM} \mathrm{NaCl}, 2,5 \mathrm{mM} \mathrm{CaCl}_{2}, 10 \mathrm{mM} \mathrm{HEPES} / \mathrm{NaOH}, \mathrm{pH} \mathrm{7,4).}$ Cell suspension was diluted to $300 \mu$ l with the corresponding buffer and analyzed by flow cytometry.

\section{Cell proliferation assays}

hMSC were harvested in Alpha MEM complete medium in 96-well flat-bottom plates at a concentration of 5 $\times 10^{3}$ cells/well and were incubated for $24 \mathrm{~h}$ before the experiment was performed. After that, complete medium was removed and cells were incubated for another $24 \mathrm{~h}$ in Alpha MEM (w/o serum) before the experiment was performed. Then, sTRAIL $(10-1000 \mathrm{ng} / \mathrm{ml})$ or LUVTRAIL $(10-1000 \mathrm{ng} / \mathrm{ml})$ were added to the culture wells and incubated during $24 \mathrm{~h}$ more. In parallel, cells were also cultured in the presence of FBS $(10 \% \mathrm{v} / \mathrm{v})$ with or without the blocking anti-human TRAIL antibody RIK2 (BD Biosciences). Finally, cell growth was analyzed by uptake of 5-bromo-2'-deoxyuridine (BrdU) of living cells and measuring of color absorbance, using the BrdU Cell Proliferation Assay Kit (Cell Signaling), following manufacturer's instructions.

\section{Immunoblot analysis}

TRAIL expression from ultracentrifugation supernatants or reconstituted pellet or from hMSC and Jurkat cell extracts was determined by immunoblot. In the latter case, $5 \times 10^{6}$ cells were collected and lysed in 50 $\mathrm{mM}$ Tris/ $\mathrm{HCl} \mathrm{pH} 7.4$ buffer containing $0,15 \mathrm{M} \mathrm{NaCl}, 10 \%$ glycerol, $1 \mathrm{mM}$ EDTA, $10 \mathrm{mg} / \mathrm{ml}$ leupeptin, $1 \mathrm{mM}$ PMSF and $1 \%$ Triton $X-100$. Then, all samples were resolved by SDS-12\% PAGE, transferred to PVDF membranes and blocked with TBS-T buffer (10 mM Tris/HCl pH 8,0: 0,12 $\mathrm{M} \mathrm{NaCl}$ : 0,1\% Tween-20) containing 5\% skimmed milk. PVDF membranes were incubated with the rabbit anti-human TRAIL antibody C92B9 (Cell Signaling) in TBS-T containing $2 \%$ skimmed milk. Membranes were washed with TBS-T and incubated with $0.2 \mu \mathrm{g} / \mathrm{ml}$ of the corresponding peroxidase-labeled secondary antibody (Sigma) and revealed with the Pierce ECL Western Blotting Substrate (Thermo Scientific).

\section{Statistical analysis}

GraphPad Prism 6 software was used to analyze computer-based statistical analysis. Results showed indicate the mean $\pm S D$ of at least two (for the cytotoxic assays) or three (for the BrdU assays) different experiments. Student's $t$ test for paired variants was performed to evaluate statistical significance. A $p<0.05$ value was considered to be significant. 

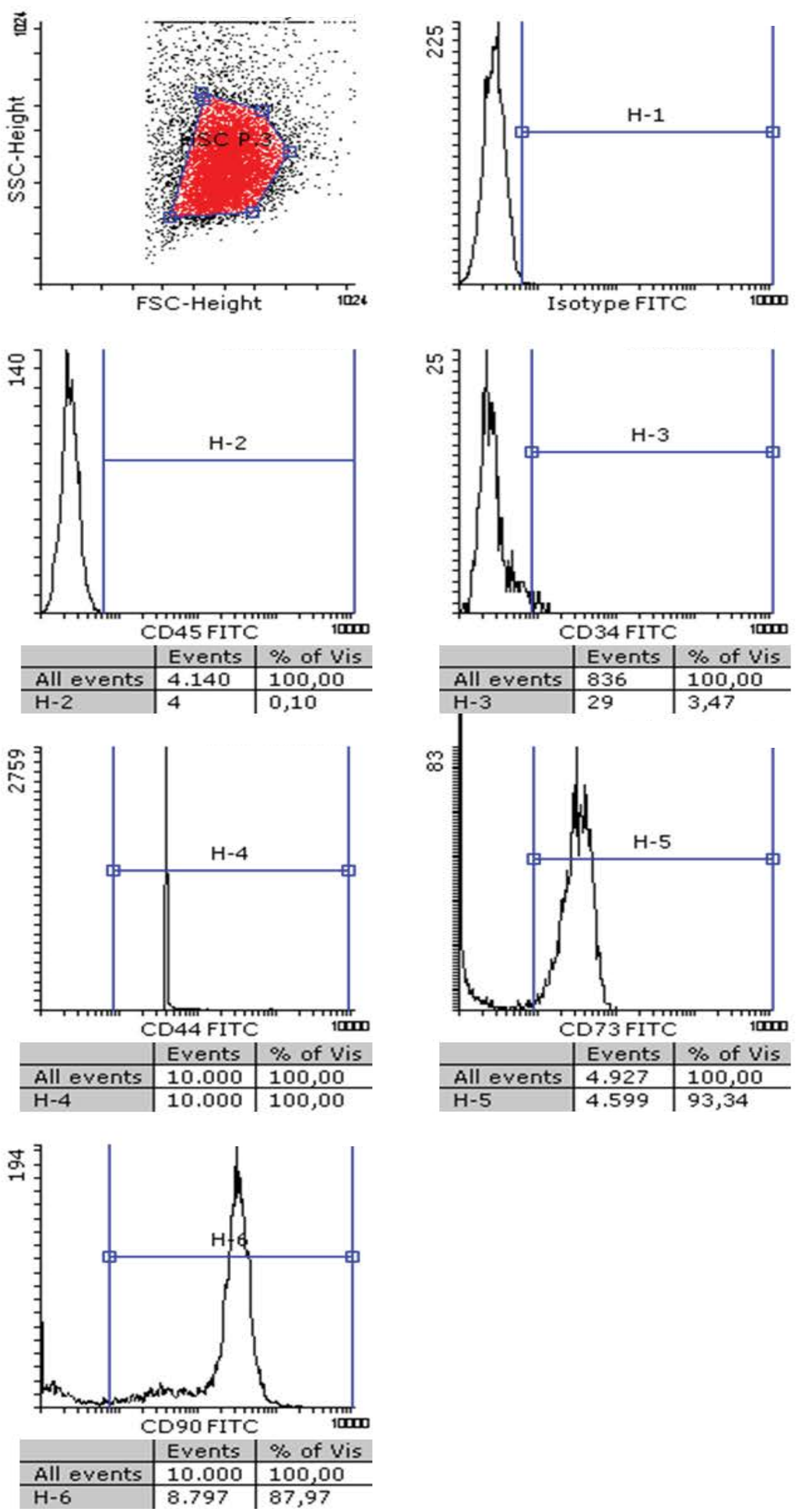

Figure 1: Characterization of hMSC: hMSC were phenotyped using flow cytometry following the Bourin consensus guidelines. Cells were incubated with an isotype control antibody labeled with FITC (upper right panel), or with specific mAbs against human CD45, CD34, CD44, CD73 or CD90, as indicated, labeled with the same fluorophore. The cell population was gated in the flow cytometer (upper left panel) and fluorescence detected in each sample. 


\section{Results}

\section{Analysis of TRAIL receptor expression on isolated hMSC}

After isolating hMSC from adipose tissue of healthy donors, a standard characterization was performed following the Bourin consensus guidelines [34]. As shown in Figure 1, isolated hMSC cells were negative for CD45 and CD34 surface expression and positive for CD44, CD73 and CD90. Afterwards, we demonstrated that these cells are able to grow similarly in the presence of $5 \%$ human platelet lysate or in the presence of $10 \%$ fetal bovine serum, duplicating in $48 \mathrm{~h}$ in both cases. Next, we explored the pattern of expression of TRAIL receptors in hMSC. As shown in Figure 2, hMSC do not express DR4 or the decoy receptors DcR1 or DcR2, but they express DR5 at a high level. Since some of the experiments to be performed in these cells were done in serum-free medium, we first determined that cells did not die after $48 \mathrm{~h}$ of serum deprivation, although their growth was stopped, and that this change of medium did not affect to TRAIL receptor expression, as also shown in Figure 2.

\section{TRAIL does not induce cell death of hMSC, whether soluble or associated with liposomes (LUV-TRAIL)}

Then, we analyzed if soluble TRAIL or the more bioactive TRAIL formulation LUV-TRAIL (TRAIL bound to large unilamellar vesicles) were able to induce apoptotic cell death on hMSC.

For that, we analyzed PS exposure on the outer leaflet of the plasma membrane by annexin-V-FITC labelling and flow cytometry, and loss of plasmas membrane integrity by labelling with 7-AAD. As shown in Figure 3 recombinant soluble TRAIL (sT) or TRAIL associated with liposomes (LUV-TRAIL, LT) did not induce apoptotic cell death in a range of concentrations from 10 to $1000 \mathrm{ng} /$ $\mathrm{ml}$, concentrations that readily induce cell death in a wide variety of tumor cells, including cells that are resistant to chemotherapeutic drugs in the case of LUVTRAIL [25]. In these experiments we also included the blocking anti-TRAIL antibody RIK-2, which was also without effect.

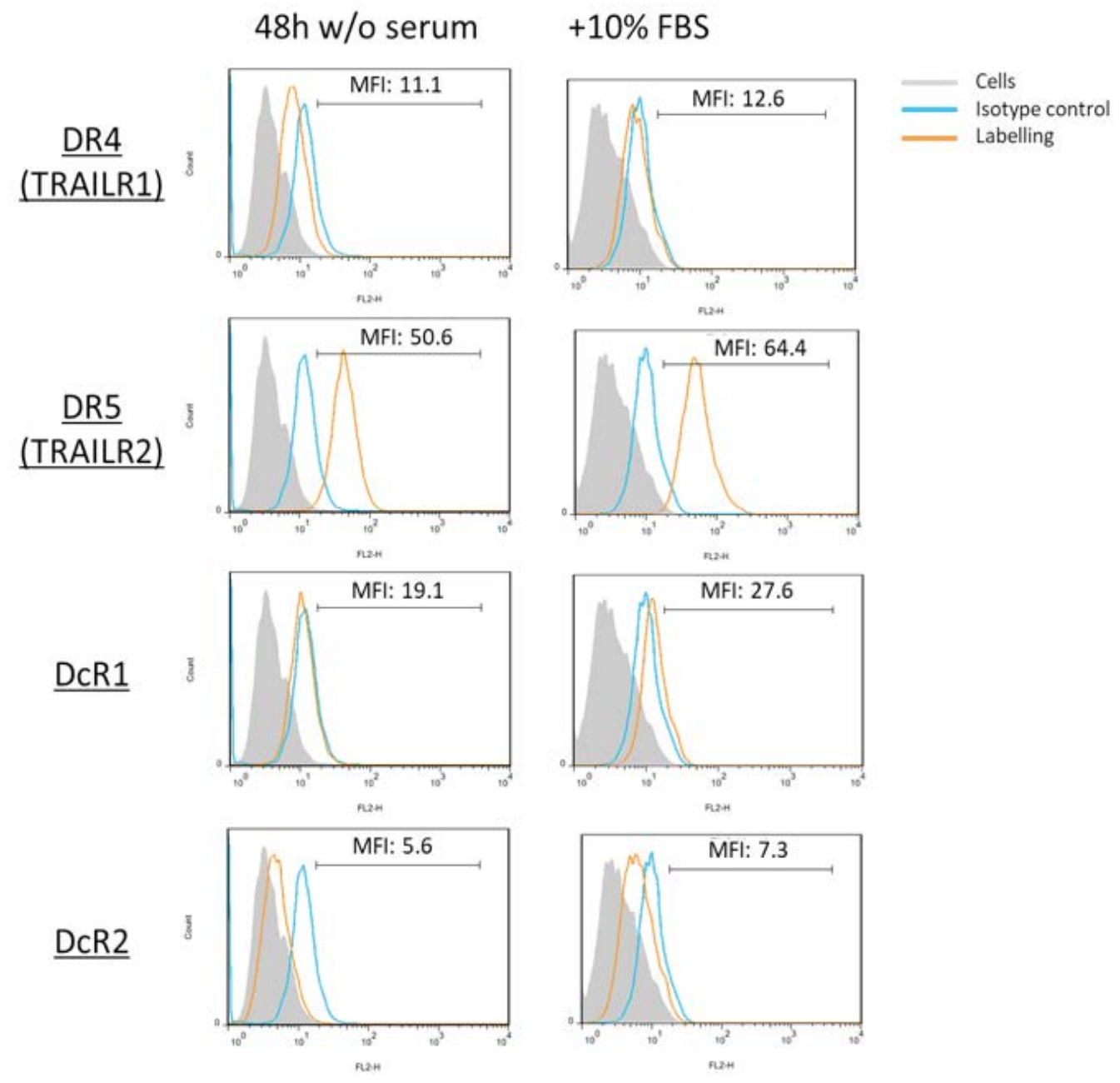

Figure 2: TRAIL receptor expression in hMSC: The expression of TRAIL receptors DR4 (TRAILR1), DR5 (TRAILR2), DcR1 and DcR2 on the surface of hMSC cells was determined by flow cytometry using specific antibodies conjugated with PE. The analysis was performed on cells cultured in the presence of $10 \%$ fetal bovine serum (right panels) or after $48 \mathrm{~h}$ of culture in medium without serum (left panels). Grey histograms, labeling of the cells alone; blue histograms, labeling with an irrelevant antibody of the same isotype and conjugated with PE; orange histograms, labeling with the anti-receptor antibodies conjugated with PE. 
TRAIL does not induce differentiation or proliferation of hMSC, whether soluble or associated with liposomes (LUV-TRAIL)

Then, we tested the possibility that TRAIL induced hMSC differentiation to some of their described cellular fates, such as osteoclasts, adipocytes, neuronal-like cells.or chondrocytes. These differentiation processes are clearly distinguished by cell morphological changes that can be explored by microscopy. For example, from their initial fibroblastic appearance, differentiation to osteoclasts is characterized by size increase, cell grouping and a "foamy" cytoplasm; differentiation to adipocytes would be characterized by the appearance of lipid droplet inclusions in the cytoplasm; and differentiation to neuronal cells by the appearance of cytoplasmic extensions in the form of dendrites [35].

Differentiation to chondrocytes is characterized by rounded morphologic appearance with cell clusters after two days in culture [36]. To perform these experiments, hMSC were cultured in serum-free medium during $48 \mathrm{~h}$ and exposed to different concentrations of soluble TRAIL or LUV-TRAIL. In parallel, cells were also cultured during the same time with $10 \%$ FBS in the presence or in the absence of the blocking anti-TRAIL antibody RIK-2. As shown in Figure 4, no change in cell morphology was observed in any experimental condition, apart from the monolayer growth and almost confluence observed in the presence of serum, which was not observed in its absence.

\section{Endogenous TRAIL contributes to the survival and growth of hMSC}

Next, we tested if STRAIL or LUV-TRAIL were able to induce the proliferation of hMSC. For that, the experiments were performed using the BdrU assay, in the absence of serum, and using cultures with $10 \%$ FBS as additional controls. As shown in Figure 5, no increase in the proliferation of hMSC was observed when cells were treated with different concentrations of recombinant TRAIL, soluble or associated with liposomes. Culture of hMSC with $10 \%$ FBS clearly induced cell growth, with cell duplication after $24 \mathrm{~h}$ of culture.

Remarkably, culture in these conditions and in the presence of the blocking anti-TRAIL mAb RIK-2 resulted in a significant inhibition of cell growth, accounting for a $50 \%$ inhibition in average. RIK-2 is a specific mouse $\mathrm{mAb}$ directed against an extracellular epitope of human TRAIL, which does not cross-react with TRAIL from other species. This result indicates that, although exogenous TRAIL is not able to support hMSC cell growth by itself, in the presence of serum, endogenous TRAIL seems to contribute to cell growth in synergy to growth factors present in serum. We also tested if exogenous STRAIL or LUV-TRAIL induced additional cell growth in the presence of serum, but did not find any effect (data

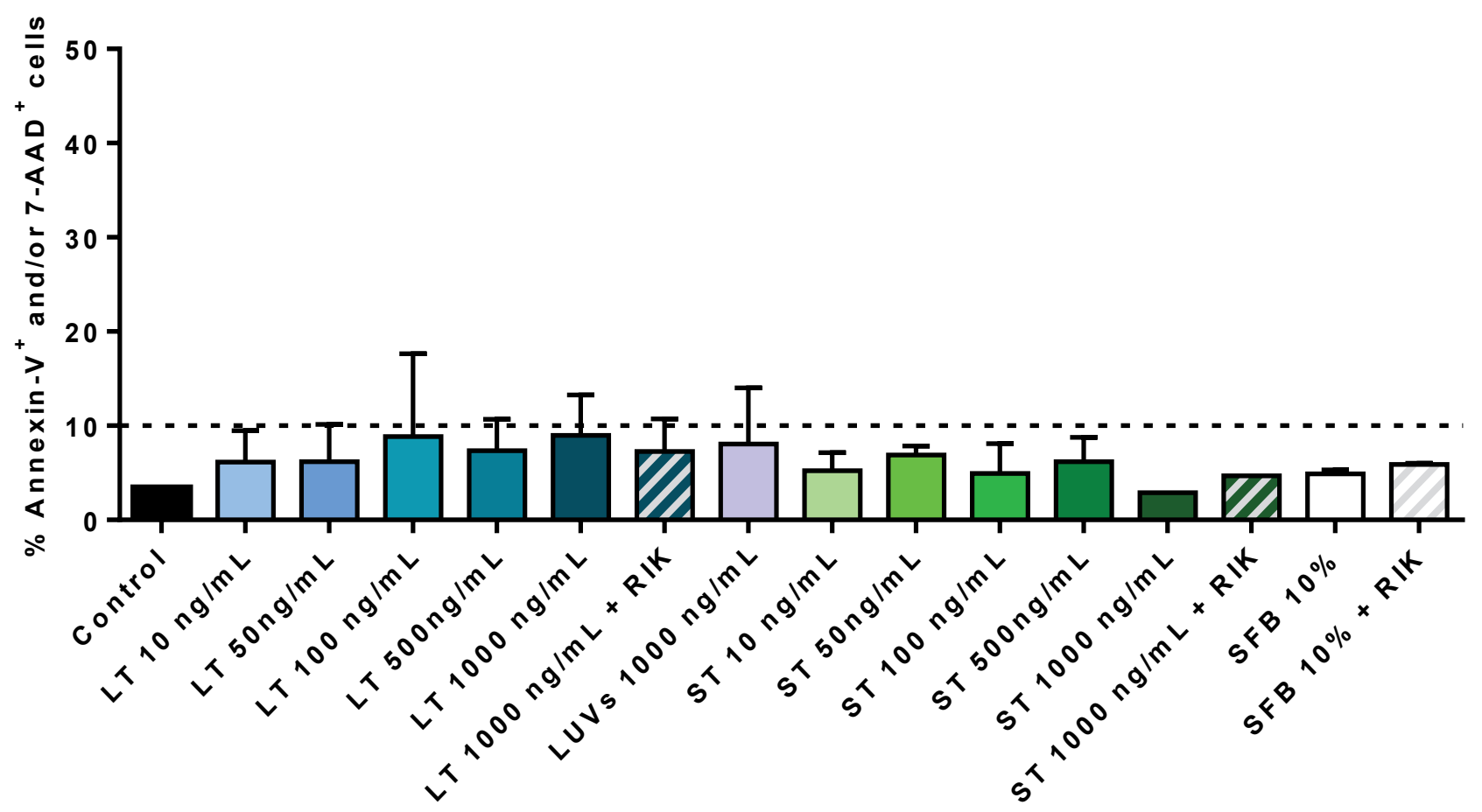

Figure 3: TRAIL does not induce cell death on hMSC: Cells were incubated or not (Control) during $24 \mathrm{~h}$ with the indicated concentrations of LUV-TRAIL (LT) or soluble TRAIL (ST) in serum-free medium. Cells were also incubated with LUVs alone or with the maximal concentrations of LT or ST in the presence of $2.5 \mu \mathrm{g} / \mathrm{ml}$ of the blocking anti-TRAIL antibody RIK2, as indicated. As an additional control, cells were also cultured in medium with $10 \%$ FBS in the presence or absence of RIK2. Then, cells were stained with annexin-V-FITC and 7-AAD and cell death analyzed by flow cytometry. Results are the mean $\pm \mathrm{SD}$ of three different experiments. 


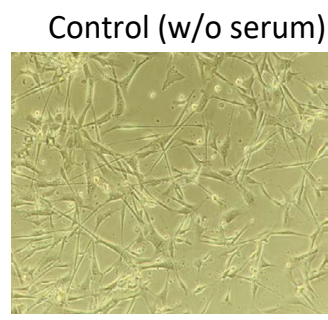

$1 \mathrm{ng} / \mathrm{mL}$
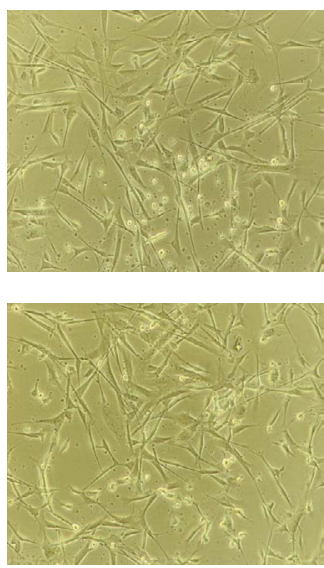

+SFB $10 \%$

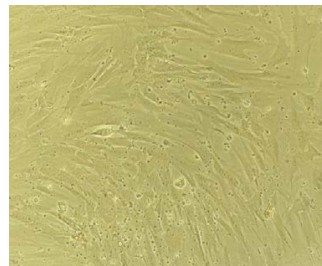

$2 \mathrm{ng} / \mathrm{mL}$
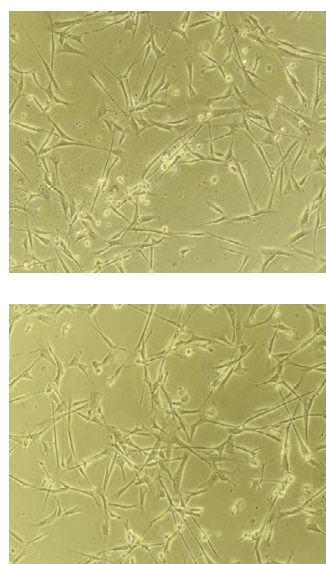

+ SFB $10 \%+R I K 1$

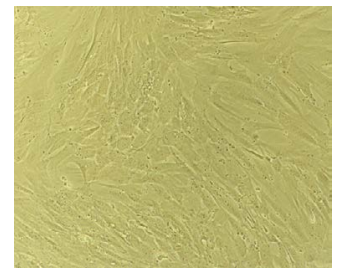

$5 \mathrm{ng} / \mathrm{mL}$
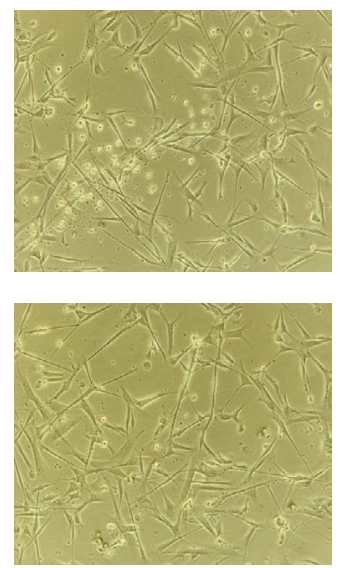

Figure 4: Morphology of hMSC treated with sTRAIL or with LUV-TRAIL: Cells were incubated or not (Control) during $24 \mathrm{~h}$ with the indicated concentrations of LUV-TRAIL or soluble TRAIL (sTRAIL) in serum-free medium. As an additional control, cells were also cultured in medium with $10 \%$ FBS in the presence or absence of RIK2. Then, representative phase contrast micrographs were obtained of each culture.

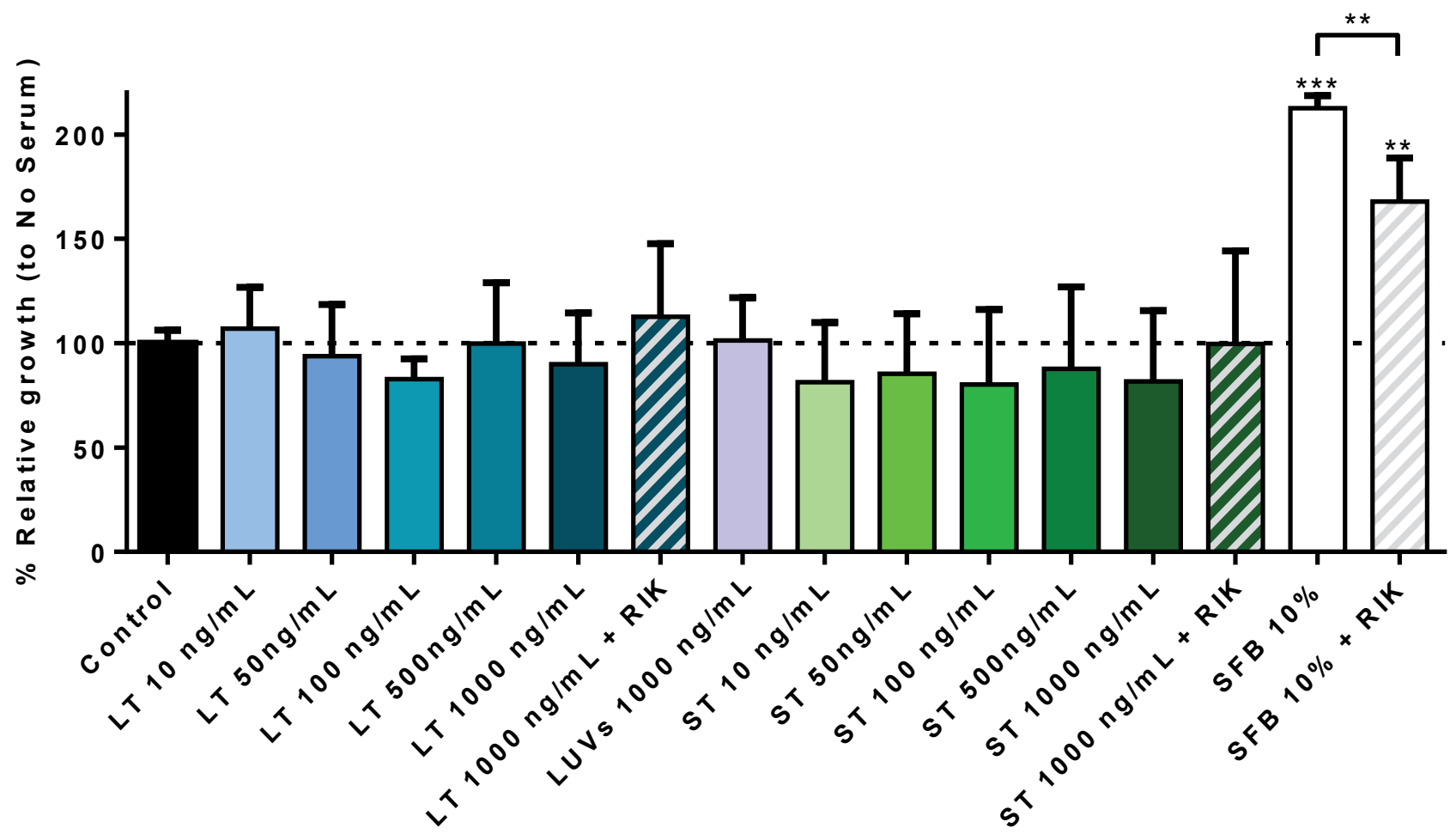

Figure 5: hMSC growth in the presence of sTRAIL or LUV-TRAIL: Cells were incubated or not (Control) during 24h with the indicated concentrations of LUV-TRAIL (LT) or soluble TRAIL (ST) in serum-free medium. Cells were also incubated with LUVs alone or with the maximal concentrations of LT or ST in the presence of $2.5 \mu \mathrm{g} / \mathrm{ml}$ of the blocking anti-TRAIL antibody RIK2, as indicated. As an additional control, cells were also cultured in medium with $10 \%$ FBS in the presence or absence of RIK2. Then, cell growth was estimated using a BrdU Cell Proliferation Assay Kit, as described in Materials and Methods. Results are the mean \pm SD of six different experiments.

${ }^{* *} P<0.02:{ }^{* *} P<0.01$. 
not shown), indicating that the contribution of TRAIL to hMSC growth is already achieved by endogenous TRAIL.

\section{TRAIL is secreted in soluble form from hMSC, but} not associated with exosomes

Next, we tested by immunoblot if hMSC did express endogenous TRAIL, comparing with the acute leukemia T cell line Jurkat, in which TRAIL expression has been characterized in previous studies [24,37]. We used a rabbit anti-TRAIL antibody validated for immunoblot purposes. As shown in Figure 6A, right panel, several isoforms of TRAIL are expressed in Jurkat cells. These different isoforms correspond to different glycosylated forms of the protein, with the mature, fully glycosylated form migrating at a molecular weight of $41-42 \mathrm{kDa}$, while the polypeptide accounted for a band around [32-35] kDa. The soluble form of the protein appears as a doublet around 23-25 kDa, with a synthesis intermediate isoform at 30 $\mathrm{kDa}$. HMSC were also positive for TRAIL expression, although at a lower level than Jurkat cells, and with the polypeptide and soluble forms predominating over the other isoforms (squared in red). We also tested TRAIL expression in hMSC by flow cytometry, showing some surface expression (blue histogram in Figure 6B), but with a higher labeling when cells were permeabilized prior to labeling (red histogram in Figure 6B). These data demonstrate that in hMSC, basal intracellular TRAIL expression is higher than TRAIL expression on the cell surface, as it is also the case in activated human T cells $[24,37]$.

If endogenous TRAIL is able to cooperate with growth factors present in the serum to support hMSC growth, it should be secreted to the supernatant. TRAIL can be secreted in association with exosomes from different cell types, including activated human $T$ cell blasts and Jurkat cells, but it can also be secreted in soluble form in certain cell types. The molecular form in which TRAIL is secreted from hMSC was tested by immunoblot. For that, we took supernatants from the cultures, and submitted them to clearing and further ultracentrifugation, concentrating the pellet by 500 -fold. We used as control of specificity the same ultracentrifugation supernatants and concentrates from culture medium in the presence or absence of FBS. As it can be observed in Figure 6A, left panel, only a faint band of the $25 \mathrm{kDa}$ soluble TRAIL was observed in the ultracentrifugation pellet from hMSC culture. This band was much more intense in the ultracentrifugation supernatant, demonstrating that TRAIL is secreted from hMSC in soluble form. The transmembrane forms of the protein were not detected in
A

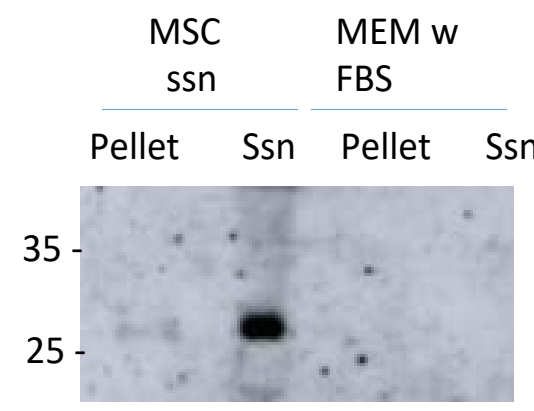

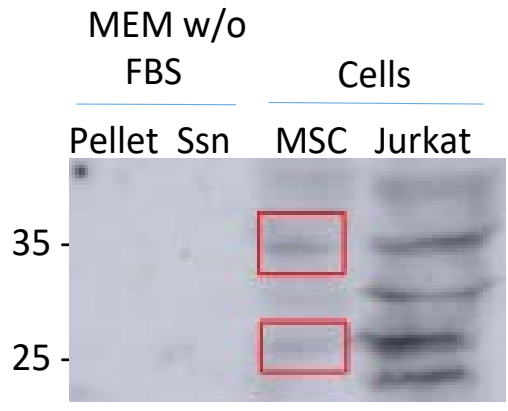

Glycosylated TRAIL

TRAIL (polypeptide)

TRAIL (intermediate)

Soluble TRAIL (doublet)

B

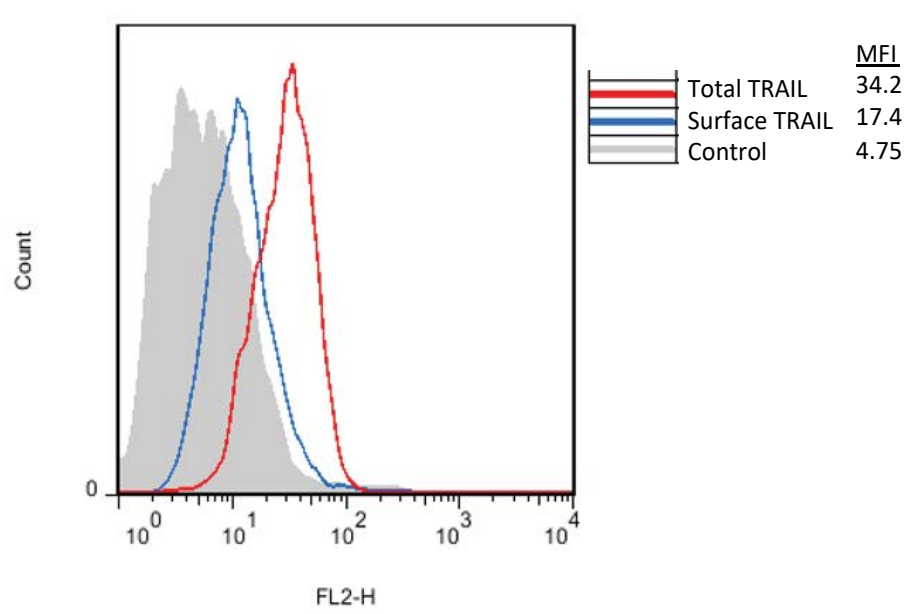

Figure 6: TRAIL detection in hMSC or in their supernatants: (A) TRAIL was detected by immunoblot on extracts from MSC or from Jurkat cells (right panel), as indicated, or on supernatants from MSC cultures subjected to ultracentrifugation, analyzing separately the ultracentrifugation pellet of its supernatant (left panel), as indicated in Materials and Methods. As controls, the same procedures were applied to culture media with (left panel) or without FBS (right panel). (B) TRAIL was detected by flow cytometry on hMSC permeabilized or not, to differentiate TRAIL expressed on the cell surface (blue histogram) or total cellular TRAIL (red histogram). The grey histogram corresponds to the labelling of the cells alone. 
the concentrated pellet ultracentrifugate, in contrast to that reported in activated human T cells or Jurkat cells $[24,38]$. No band could be detected in the ultracentrifugation pellet or supernatant of the control culture media that had not been in contact with the cells, whether FBS was present or not (Figure 6).

\section{Discussion}

Although hMSC transfected with TRAIL have shown promising pre-clinical efficacy in tumors, especially against gliomas $[18,20]$, little was known about TRAIL bioactivity on hMSC cells themselves, whether it was able to induce cell death, or rather differentiation or proliferation. In the present work, we have isolated adipose tissue-derived hMSC from healthy donors and analyzed the effects of sTRAIL or LUV-TRAIL on them. Results showed that TRAIL by itself does not affect hMSC viability or promote differentiation on these primary cells. It is important to point out that the increase in receptor oligomerization and the corresponding pro-apoptotic signaling induced by the attachment of TRAIL to liposomes (LUV-TRAIL), clearly demonstrated in many cancer types [25-28], has no effect on hMSC, indicating that these cells are very well protected against apoptotic cell death. Previous data indicated that soluble TRAIL or agonistic anti-TRAIL receptor antibodies induced proliferation of a prediapocyte cellular model derived from a Simpson-Golabi-Behmel Syndrome (SGBS) patient, resulting also in an inflammatory response [16,17]. In those studies, TRAIL did not induce apoptosis or differentiation in this cellular model, in line with our results. The fact that they used preadipocytes from a different origin (SGBS cell line) could explain the apparent discordant results. In another model of hMSC derived from bone marrow, Secchiero, et al. did not detect cell death, nor proliferation or differentiation induced by soluble TRAIL, but rather migration in transwell plates [15].

In fact, our study points out to a positive proliferative effect of endogenous TRAIL on hMSC, but it seems that it should act in synergy with growth factors present in FBS used in the in vitro culture. This proliferative effect was mediated by DR5, given that the expression of the other transmembrane receptors could not be found in our cultured hMSC cells. DR5 expression has been reported previously in preadipocytes [39], as well as in bone marrow-derived MSC [15]. The proliferative role of TRAIL has been described both in normal and tumor cells [40].

It is not fully understood either whether TRAIL is secreted or not in association with exosomes in TRAIL-expressing cells. TRAIL is expressed in many types of immune cells, such as T cells, NK cells, dendritic cells and macrophages. TRAIL is secreted bound to exosomes during the process of activation-induced cell death in human $T$ cells, contributing to immune regulatory processes $[23,24]$. It has also been described its secretion in association with microvesicles by tumor cells, in order to suppress anti-tumor T lymphocyte action [41-43] and by placenta, as an immune protective mechanism [44].

In this work, we found out that endogenous TRAIL from hMSC would be exerting its function in soluble form, as TRAIL was not detected in hMSC-derived exosomes. It has been described that when MSC are transfected to overexpress TRAIL, this protein can be found in secreted microvesicles [45] although other studies performed in bone marrow-derived MSC transfected with full-length or soluble TRAIL have demonstrated that these cells secrete TRAIL only in its soluble form [46]. Therefore, it seems that TRAIL secretion in association with exosomes could be dependent either on the cell type or on its expression level.

FasL and TRAIL present a cleavage site that would release soluble fragments of $27 \mathrm{kDa}$ and of $24 \mathrm{kDa}$, respectively. In the case of FasL, the shedding of soluble FasL has been attributed to the metalloprotease ADAM10 [47]. Regarding TRAIL, it has been described that metalloproteinase 2 (MMP2) cleaves the recombinant molecule in vitro [48]. However, this matter still remains controversial since in another study, the metalloproteinase inhibitor 1,10-phenanthroline did not block in vitro recombinant TRAIL processing and it was suggested that it was rather processed by a still undefined cysteine protease [49]. In any case, no evidence of TRAIL cleavage by metalloproteinases has been found in vivo.

As a conclusion, in this work, we demonstrate that exogenous TRAIL does not affect growth, viability or differentiation of hMSC, either in soluble form or in its higher bioactive form, bound to liposomes. Nevertheless, we also show that endogenous TRAIL from hMSC, which was secreted in soluble form and not in association with exosomes, did affect positively their proliferation. The present data allows a better knowledge of hMSC biology and points to a positive role of TRAIL in hMSC survival and proliferation. This could be important if hMSC transfected with TRAIL are used against tumor development, since secreted TRAIL could exert its anti-tumoral effect at the same time as hMSC would persist. On the other hand, if these cells are used for regenerative medicine purposes, such as articular regeneration, TRAIL produced by the same hMSC or by surrounding immune cells, would contribute to their persistence and engrafting.

\section{Acknowledgements}

We greatly acknowledge Juan Carlos de Gregorio from Peaches Biotech and Eduardo Almenara, from OTRI office from University of Zaragoza, for their help in the establishment of contracts for funding this research.

\section{Conflict of Interest}

All authors concur with the submission the work has not been published elsewhere and has not been submitted to another journal.

The authors declare no conflict of interest. 


\section{Funding}

This work was supported by a contract established by Peaches Biotech Group with OTRI from University of Zaragoza.

\section{References}

1. Pitti RM, Marsters SA, Ruppert S, Donahue CJ, Moore A, et al. (1996) Induction of apoptosis by apo-2 ligand, a new member of the tumor necrosis factor cytokine family. $\mathrm{J}$ Biol Chem 271: 12687-12690.

2. Wiley SR, Schooley K, Smolak PJ, Din WS, Huang CP, et al. (1995) Identification and characterization of a new member of the tnf family that induces apoptosis. Immunity 3 : 673-682.

3. Naval J, Miguel D, Gallego-Lleyda A, Anel A, Martinez-Lostao L, et al. (2019) Importance of TRAIL Molecular Anatomy in Receptor Oligomerization and Signaling. Implications for Cancer Therapy. Cancers 11: 444.

4. Jouan-Lanhouet S, Arshad M, Piquet-Pellorce C, Martin-Chouly C, Moigne-Muller G, et al. (2012) TRAIL induces necroptosis involving RIPK1/RIPK3-dependent PARP-1 activation. Cell Death Differ 19: 2003-2014.

5. Almasan A, Ashkenazi A (2003) Apo2L/TRAIL: Apoptosis signaling, biology, and potential for cancer therapy. Cytokine Growth Factor Rev 14: 337-348.

6. Hymowitz SG, Christinger M, Fuh G, Ultsch M, O'Connell $M$, et al. (1999) Triggering cell death: The crystal structure of Apo2L/TRAIL in a complex with death receptor 5 . Mol Cell 4: 563-571.

7. LeBlanc HN, Ashkenazi A (2003) Apo2L/TRAIL and its death and decoy receptors. Cell Death Differ 10: 66-75.

8. Ehrhardt H, Fulda S, Schmid I, Hiscott J, Debatin K-M, et al. (2003) Induced survival and proliferation in cancer cells resistant towards TRAIL-induced apoptosis mediated by NF-kappaB. Oncogene 22: 3842-3852.

9. Jeremias I, Kupatt C, Baumann B, Herr I, Wirth T, et al. (1998) Inhibition of nuclear factor kappaB activation attenuates apoptosis resistance in lymphoid cells. Blood 91: 46244631.

10. Secchiero P, Gonelli A, Carnevale E, Milani D, Pandolfi A, et al. (2003) TRAIL promotes the survival and proliferation of primary human vascular endothelial cells by activating the Akt and ERK pathways. Circulation 107: 2250-2256.

11. Secchiero $P$, Melloni E, Heikinheimo M, Mannisto S, Pietro $R$, et al. (2004) TRAIL regulates normal erythroid maturation through an ERK-dependent pathway. Blood 103: 517-522.

12. Bartolo B, Cartland S, Prado-Lourenco L, Griffith TS, Gentile C, et al. (2015) Tumor Necrosis Factor-Related Apoptosis-Inducing Ligand (TRAIL) promotes angiogenesis and ischemia-induced neovascularization via NADPH oxidase 4 (NOX4) and nitric oxide-dependent mechanisms. J Am Heart Assoc 16: e002527.

13. Kavurma M, Schoppet M, Bobryshev Y, Khachigian LM, Bennett MR, et al. (2008) TRAIL stimulates proliferation of vascular smooth muscle cells via activation of NF-kappaB and induction of insulin-like growth factor-1 receptor. J Biol Chem 283: 7754-7762.

14. Herrero-Martin G, Hoyer-Hansen M, Garcia-Garcia C, Fumarola C, Farkas T, et al. (2009) TAK1 activates AMPK-dependent cytoprotective autophagy in TRAIL-treated epithelial cells. EMBO J 28: 677-685.
15. Secchiero P, Melloni E, Corallini F, Beltrami AP, Alviano F, et al. (2008) Tumor necrosis factor-related apoptosis-inducing ligand promotes migration of human bone marrow multipotent stromal cells. Stem Cells 26: 2955-2963.

16. Funcke J, Zoller V, Hay M, Debatin K-M, Wabitsch M, et al. (2015) TNF-related apoptosis-inducing ligand promotes human preadipocyte proliferation via ERK1/2 activation. FASEB J 29: 3065-3075.

17. Zoller V, Funcke J, Roos J, Dahlhaus M, Hay MA, et al. (2017) TNF-related apoptosis-inducing ligand induces an inflammatory response in human adipocytes. Sci Rep 7: 5691.

18. Loebinger M, Eddaoudi A, Davies D, Janes SM (2009) Mesenchymal stem cell delivery of trail can eliminate metastatic cancer. Cancer Res 69: 4134-4142.

19. Mueller L, Luetzkendorf J, Widder M, Nerger K, Caysa H, et al. (2011) TRAIL-transduced multipotent mesenchymal stromal cells (TRAIL-MSC) overcome TRAIL resistance in selected CRC cell lines in vitro and in vivo. Cancer Gene Ther 18: 229-239.

20. Kim SM, Lim JY, Park SI, Jeong CH, Oh JH, et al. (2008) Gene therapy using TRAIL-secreting human umbilical cord blood-derived mesenchymal stem cells against intracranial glioma. Cancer Res 68: 9614-9623.

21. Kim SM, Oh JH, Park SA, Ryu CH, Lim JY, et al. (2010) Irradiation enhances the tumor tropism and therapeutic potential of tumor necrosis factor-related apoptosis-inducing ligand-secreting human umbilical cord blood-derived mesenchymal stem cells in glioma therapy. Stem Cells 28: 2217-2228

22. Han H, Park S, Ahn S, Jeun S-S, Ryu CH (2019) Evaluation of combination treatment effect with TRAIL-secreting mesenchymal stem cells and compound $C$ against glioblastoma. Anticancer Res 39: 6635-6643.

23. Anel A, Gallego-Lleyda A, Miguel D, Naval J, MartínezLostao $L$ (2019) Role of exosomes in the regulation of t-cell mediated immune responses and in autoimmune disease. Cells 8: 154.

24. Martínez-Lorenzo MJ, Anel A, Gamen S, Monle I, Lasierra $P$, et al. (1999) Activated human T Cells release bioactive fas ligand and APO2 ligand in microvesicles. J Immunol 163: 1274-1281.

25. Miguel D, Basáñez G, Sánchez D, Malo PG, Marzo I, et al. (2013) Liposomes decorated with Apo2L/TRAIL overcome chemoresistance of human hematologic tumor cells. Mol Pharm 10: 893-904.

26. Gallego-Lleyda A, Miguel D, Anel A, Martinez-Lostao L (2018) Lipid nanoparticles decorated with TNF-related aptosis-inducing ligand (TRAIL) are more cytotoxic than soluble recombinant trail in sarcoma. Int J Mol Sci 19: E1449.

27. Miguel D, Gallego-Lleyda A, Ayuso J, Pawlak A, Conde B, et al. (2016) Improved anti-tumor activity of novel highly bioactive liposome-bound TRAIL in breast cancer cells. Recent Pat Anticancer Drug Discover 11: 197-214.

28. Miguel S, Gallego-Lleyda A, Ayuso JM, Pejenaute-Ochoa D, Jarauta V, et al. (2016) High-order TRAIL oligomer formation in trail-coated lipid nanoparticles enhances dr5 cross-linking and increases antitumour effect against colon cancer. Cancer Lett 383: 250-260.

29. Miguel D, Gallego-Lleyda A, Martinez-Ara M, Plou J, Anel A, et al. (2019) Double-edged lipid nanoparticles combining liposome-bound trail and encapsulated doxorubicin showing an extraordinary synergistic pro-apoptotic potential. Cancers 11: E1948. 
30. Elahi F, Farwell D, Nolta J, Anderson JD (2020) Preclinical translation of exosomes derived from mesenchymal stem/ stromal cells. Stem Cells 38: 15-21.

31. Martínez-Lorenzo M, Royo-Cañas M, Alegre-Aguarón E, Desportes P, Castiella T, et al. (2009) Phenotype and chondrogenic differentiation of mesenchymal cells from adipose tissue of different species. J Orthop Res 27: 1499-1507.

32. Martinez-Lostao L, García-Alvarez F, Basáñez G, AlegreAguarón E, Desportes P, et al. (2010) Liposome-bound APO2L/TRAIL is an effective treatment in a rabbit model of rheumatoid arthritis. Arthrit Rheum 62: 2272-2282.

33. MacFarlane M, Ahmad M, Srinivasula S, Fernandes-Alnemri T, Cohen GM, et al. (1997) Identification and molecular cloning of two novel receptors for the cytotoxic ligand TRAIL. J Biol Chem 272: 25417-25420.

34. Bourin P, Bunnell B, Cateilla L, Dominici M, Katz AJ, et al. (2013) Stromal cells from the adipose tissue-derived stromal vascular fraction and culture expanded adipose tissue-derived stromal/stem cells: A joint statement of the international federation for adipose therapeutics (IFATS) and science and the international society for cellular therapy (ISCT). Cytotherapy 15: 641-648.

35. Hanna H, Mir L, Andre F (2018) In vitro osteoblastic differentiation of mesenchymal stem cells generates cell layers with distinct properties. Stem Cell Res Ther 9: 203.

36. Worster A, Brower-Toland B, Fortier L, Bent SJ, Williams J, et al. (2001) Chondrocytic differentiation of mesenchymal stem cells sequentially exposed to transforming growth factor-beta1 in monolayer and insulin-like growth factor-I in a three-dimensional matrix. J Orthop Res 19: 738-749.

37. Martínez-Lorenzo MJ, Alava MA, Gamen S, Kim KJ, Chuntharapai A, et al. (1998) Involvement of apo2 ligand/ trail in activation-induced death of jurkat and human peripheral blood t cells. Eur J Immunol 28: 2714-2725.

38. Monleón I, Martínez-Lorenzo MJ, Monteagudo L, Lasierra $P$, Taulés $M$, et al. (2001) Differential secretion of fas ligand- or apo2 ligand/trail-carrying microvesicles during activation-induced death of human T cells. J Immunol 167: 6736-6744.

39. Keuper M, Asterholm IW, Scherer P, Westhoff M-A, Möller P, et al. (2013) TRAIL TNF-related apoptosis-inducing li- gandregulates adipocyte metabolism by caspase-mediated cleavage of PPARgamma. Cell Death Dis 4: 474.

40. Lafont E, Hartwig T, Walczak H (2018) Paving TRAIL's path with ubiquitin. Trends Biochem Sci 43: 44-60.

41. Huber V, Fais S, lero M, Lugini L, Canese P, et al. (2005) Human colorectal cancer cells induce T-cell death through release of proapoptotic microvesicles: Role in immune escape. Gastroenterol 128: 1796-1804.

42. Martínez-Lorenzo MJ, Anel A, Alava MA, Piñeiro A, Naval $J$, et al. (2004) The human melanoma cell line MelJuso secretes bioactive FasL and APO2L/TRAIL on the surface of microvesicles Possible contribution to tumor counterattack. Exp Cell Res 295: 315-329.

43. Valenti $R$, Huber V, lero M, Filipazzi P, Parmiani G, et al. (2007) Tumor-released microvesicles as vehicles of immunosupression. Cancer Res 67: 2912-2915.

44. Stenqvist A-C, Nagaeva O, Baranov V, Mincheva-Nilsson L (2013) Exosomes secreted by human placenta carry functional fas ligand and TRAIL molecules and convey apoptosis in activated immune cells, suggesting exosome-mediated immune privilege of the fetus. J Immunol 191: 5515-5523.

45. Yuan ZQ, Kolluri KK, Gowers KHC, Janes SM (2017) TRAIL delivery by MSC-derived extracellular vesicles is an effective anticancer therapy. J Extracell Ves 6: 1265291.

46. Mohr A, Chu T, Brooke GN, Zwacka RM (2019) MSC.s TRAIL has better efficacy than MSC.FL-TRAIL and in combination with AKTi blocks pro-metastatic cytokine production in prostate cancer cells. Cancers (Basel) 11: 568.

47. Schulte M, Reiss K, Lettau M, Maretzky T, Ludwig A, et al. (2007) ADAM10 regulates fasl cell surface expression and modulates fasl-induced cytotoxicity and activation-induced cell death. Cell Death Differ14: 1040-1049.

48. Secchiero P, Gonelli A, Corallini F, Ceconi C, Ferrari R, et al. (2010) Metalloproteinase 2 cleaves in vitro recombinant TRAIL: Potential implications for the decreased serum levels of TRAIL after acute myocardial infarction. Atherosclerosis 211: 333-336.

49. Mariani SM, Krammer PH (1998) Differential regulation of TRAIL and CD95 ligand in transformed cells of the T and B lymphocyte lineage. Eur J Immunol 28: 973-982. 